\title{
Movilización social y deliberación \\ El marco de acción colectiva como conclusión de un diálogo deliberativo*
}

Recibido: 30/08/2019 | Revisado: 03/02/2020 | Aceptado: 05/02/2020

DOI: 10.17230/co-herencia.17.32.4

\author{
Daniel Mejía Saldarriaga** \\ dmejias2@eafit.edu.co \\ María Rocío Arango*** \\ marango@eafit.edu.co
}

\begin{abstract}
Resumen El propósito de este artículo es explicar la acción colectiva que realizan las organizaciones de movimientos sociales mediante procesos de enmarcado entendidos como el resultado de un diálogo deliberativo. Para lograrlo, el texto se divide en cuatro apartados: en el primero se introduce la teoría del enmarcado de los estudios sobre movimientos sociales. En el segundo se pasa revista sobre distintas explicaciones a propósito de la creación de un marco de acción colectiva; en el tercero se apela a la caracterización de diálogo deliberativo de Walton y Krabbe (2017) y al esquema de razonamiento práctico de Fairclough y Fairclough (2012), para trazar una relación entre el enmarcado de acción colectiva y aquel diálogo. En el último, se concluye que la acción colectiva de los activistas que se involucran en los procesos de enmarcado puede comprenderse como una acción colectiva deliberada.
\end{abstract}

\section{Palabras clave:}

Acción colectiva, marco de acción colectiva, razonamiento práctico, movimiento social, organización del movimiento social, diálogo deliberativo.

\section{Social Mobilization and Deliberation The Collective Action Frame as the Conclusion of a Deliberative Dialogue}

\footnotetext{
Abstract The purpose of this paper is to explain the collective action of social movement organizations by means of framing processes that are understood as the result of a deliberative dialogue. To this end, the text is divided into four sections. The first section introduces the
}

"Trabajo desarrollado en el marco del proyecto Contribución al desarrollo de una teoría integrada de la acción colectiva (TIAC), realizado por distintos grupos de investigación de la Universidad EAFIT durante el período 2018-2020, con la financiación de la Universidad EAFIT. $\mathrm{El}$ artículo se inscribe en el componente de estudios sobre movimientos sociales del proyecto, a cargo del grupo de investigación "Estudios en filosofía, hermenéutica y narrativas". Este componente es financiado por Colciencias gracias a la modalidad de jóvenes investigadores e innovadores (812-2018) bajo el proyecto titu- 
framing theory of social movement studies; the second section examines different explanations concerning the creation of a collective action frame. The third section considers the characterization of Walton and Krabbe's deliberative dialogue (2017) as well as Fairclough and Fairclough's practical reasoning scheme (2012) for the purpose of tracing a relationship between collective action framing and such dialogues. The last section concludes that the collective action of activists involved in the framing processes can be understood as a deliberate collective action.

\section{Keywords:}

Collective action, collective action frame, practical reasoning, social movement, social movement organization, deliberative dialogue. lado Acción colectiva en movimientos sociales constructores de paz.

** Magíster en Estudios Humanísticos de la Universidad EAFIT, Medellín, Colombia. ORCID: 0000-0003$4522-5898$

**** Profesora asociada al Departamento de Humanidades de la Universidad EAFIT, Medellín, Colombia. ORCID: 0000-00020068-9843

Según Charles Tilly y Lesley Wood (2009), uno de los errores que a menudo se cometen cuando se habla de movilización social es tratar al movimiento social como un actor unitario. Por el contrario, estos autores señalan que una campaña de movilización social vincula por lo menos a tres actores distintos: el grupo o la comunidad agraviada, el objeto de la reivindicación (autoridad pertinente) y el público que presencia e interactúa en la contienda.

De manera parecida, Scott Hunt, Robert Benford y David Snow (1994) señalan tres actores, o campos de identidad, relevantes para la movilización social: protagonistas, antagonistas y audiencias. Cada uno de estos campos cobija distintas atribuciones de identidad que se agrupan de acuerdo con objetivos particulares. Por ejemplo, con respecto a las causas defendidas por un movimiento social, los protagonistas las defienden, los antagonistas se oponen a ellas, y las audiencias, aunque en principio neutrales o no comprometidas con la causa, pueden reaccionar a favor o en contra de la campaña o del uso de repertorios de movilización.

Dentro del campo de identidad de los protagonistas se encuentran las Organizaciones del Movimiento Social (Social Movement Organization, $\mathrm{SMO}$ en adelante). Este tipo de organización es definida por Jurgen Willems y Marc Jegers (2012, p. 68) como "organizaciones formales sin fines de lucro, con fines sociales o de la sociedad civil que comparten misiones y visiones interdependientes y están asociadas 
con movimientos sociales más amplios". ${ }^{1}$ En este sentido, las $\mathrm{SMO}$ son las encargadas de determinar los objetivos de una movilización y de diseñar las estrategias y el repertorio de acciones para alcanzarlos (Morris \& Staggenborg, 2004, p. 183).

Para llevar a cabo su labor, las SMO realizan distintos tipos de acciones colectivas. A este respecto, Tilly y Wood afirman que "el grueso de los participantes divide su tiempo entre la reivindicación pública y otras actividades, como, por ejemplo, la organización cotidiana que hay detrás de una campaña" (2009, p. 22). De acuerdo con esto, es posible afirmar, al menos provisionalmente, que las SMO realizan al menos dos tipos de acciones básicas: las relacionadas con la campaña u objetivo de la movilización, y las relacionadas con la organización de la movilización social. Es decir, una serie de acciones públicas que ejecutan una campaña particular, y otra serie de acciones privadas en las que se organiza y planea tal ejecución.

Distintos autores han acogido la distinción anterior, refiriéndose a las acciones relacionadas con la organización como procesos de enmarcado. Según Hunt et al.:

[...] en el ámbito de la acción colectiva, se proponen y afirman las identidades individuales y colectivas pertinentes de dos formas analíticas distintas, pero interrelacionadas: por medio de la participación en la acción colectiva en sí misma, tales como como protestar y celebrar, y mediante procesos de enmarcado (1994, p. 186).

La apelación a los procesos de enmarcado ha sido de gran relevancia para el entendimiento de la acción colectiva. Así, para Rhys Williams (2004), la denominada teoría del enmarcado contribuye significativamente a la comprensión de la movilización social al "teorizar explícitamente sobre el trabajo simbólico y de sentido realizado por los activistas del movimiento al articular las reivindicaciones, generar consenso sobre la importancia y formas de acción colectiva que deben perseguirse, y presentar las razones de sus acciones" (2004, p. 93).

De acuerdo con lo anterior, el presente artículo se centra precisamente en el análisis de la acción colectiva relacionada con la organización que realizan las SMO mediante procesos de

1 La traducción de las citas de los textos escritos en inglés es nuestra. 
enmarcado. En concreto, el propósito es explicar tal tipo de acción colectiva apelando al diálogo deliberativo. Es decir, elucidar si el enmarcado de la acción colectiva realizado por los activistas ${ }^{2}$ a fin de organizar la movilización social puede entenderse como resultado de una deliberación. Para ello, el texto se divide en cuatro partes: en la primera se introduce la teoría de los marcos de acción colectiva; la segunda parte se ocupa de revisar explicaciones relevantes sobre los procesos de enmarcado de la acción colectiva con el fin de plantear una hipótesis inicial: un marco de acción colectiva se construye por medio de un tipo de diálogo argumentativo. En la tercera parte se desarrolla dicha hipótesis al afirmar que el proceso de enmarcado de acción colectiva se da mediante un diálogo deliberativo. Por último, la quinta parte concluye con la explicación de por qué el enmarcado de la acción colectiva puede definirse como una acción colectiva deliberada.

\section{El enmarcado de la acción colectiva}

La noción de marco, también referida como esquema o escenario, es acuñada por Gregory Bateson (1955) para explicar por qué una interpretación se centra más en algunos aspectos de la realidad que en otros. Un precedente importante de estos términos es la noción de prototipo, acuñada por Ludwig Wittgenstein (2008 [1953]) y retomada más adelante por Eleanor Rosch (1973). Tal noción se refiere a que tenemos en nuestra memoria algunos inventarios de formas de estructurar, clasificar e interpretar experiencias a los cuales podemos acceder de distintas maneras.

No obstante, la mayoría de los académicos reconoce la obra de Erving Goffman (1974) como precursora de la noción de marco. Tal como apunta Rom Harré (2006), el trabajo de Goffman sigue estando en el corazón de la revolución de la psicología social por las múltiples formas en las que sus nociones han sido apropiadas por otros autores y perspectivas. Pese a tal apropiación, se conserva la idea de que un marco es un esquema de interpretación que permite localizar, identificar, percibir y etiquetar experiencias.

2 La bibliografía sobre movimientos sociales ha utilizado distintos términos para referirse a los individuos que lideran las SMO. Los más usuales son activistas, actores, organizadores, y líderes. Para los propósitos de este trabajo, se utilizará la palabra activista para referirse a tales individuos. 
Los estudios sobre movimientos sociales hacen acopio del concepto de marco de Goffman con el fin de explicar los distintos procesos mediante los cuales una SMO planea sus acciones. Así, Snow y sus distintos colaboradores acuñan el término marco de acción colectiva para referirse, en términos generales, a los conjuntos de creencias y significados orientados a la acción que inspiran y legitiman las actividades de los movimientos sociales. Estos marcos realizan una "función interpretativa" al simplificar aspectos del mundo con la intención de movilizar a potenciales participantes y simpatizantes, ganar apoyo de los observadores y desmovilizar a los antagonistas (Snow \& Benford, 2000, p. 614).

Snow y Benford (1988) le confieren al enmarcado de la acción colectiva tres tareas básicas: el enmarcado de diagnóstico, de pronóstico y el motivacional. El primero identifica la situación problemática o motivo de la reivindicación, sus causas y los actores o autoridades responsables. El segundo articula una propuesta de solución, direcciona un plan de ataque, o establece las directrices para el diseño de las estrategias necesarias para resolver el problema identificado. Este enmarcado puede incluir también un contraenmarcado en relación con otros movimientos opositores o contrarios. El enmarcado motivacional, por su parte, proporciona la justificación para la participación en las actividades propuestas por las SMO mediante la construcción de vocabularios o relatos que ofrecen motivos para actuar.

Estos marcos de acción colectiva se transmiten mediante un conjunto de procesos superpuestos de orden discursivo, estratégico y controvertido. Los procesos discursivos son las conversaciones y comunicados que comparten los activistas respecto de sus actividades; son esenciales para la articulación de las distintas creencias y experiencias de los activistas, y para la amplificación o selección de dichas creencias y experiencias relevantes para el marco de acción colectiva.

Los procesos estratégicos se dirigen a objetivos particulares, como reclutar miembros, movilizar participantes o adquirir recursos. Este proceso se compone de cuatro subprocesos básicos: vinculación, amplificación, extensión y transformación de marcos. El primero conecta dos marcos que son congruentes ideológicamente, pero 
distintos estructuralmente, acerca de un problema. El segundo mejora, clarifica o dinamiza las creencias y los valores actuales. El tercero extiende los intereses y marcos primarios de un movimiento para incluir asuntos de presunto interés para los posibles participantes. El cuarto subproceso cambia viejos significados por otros nuevos.

Ahora bien, articular y amplificar creencias, así como reclutar miembros y movilizar participantes, no son tareas sencillas. Estos procesos son controvertidos por cuanto los activistas deben enfrentar distintos desafíos (internos y externos) para lograr los propósitos del movimiento. En primer lugar, se corre el riesgo de que el enmarcado lo realicen agentes externos a las $\mathrm{SMO}$, como sucede con los medios de comunicación o los movimientos rivales. En segundo lugar, se puede caer en disputas de marco, esto es, incurrir en desacuerdos acerca de realidades -presentes o proyectadas- planteados por alguna facción de los activistas o por SMO similares. Por último, se puede presentar un riesgo derivado de la relación dialéctica entre el marco y los eventos. Es decir, es posible que el enmarcado inicial termine transformando el significado y la estructura del discurso de manera que se limite la oportunidad de acción colectiva del movimiento.

Con el fin de lograr mayor participación y movilización, los marcos de acción colectiva pueden vincularse bajo un marco más general, que Snow y Benford (1992) denominan marco maestro de acción colectiva. Este tipo de marco realiza, a mayor escala, las tareas y procesos de un marco de acción colectiva. Es decir, "vincula otros marcos más específicos con esquemas cognitivos más amplios, que son compartidos por la mayoría de los participantes y simpatizantes del movimiento" (Diani, 1995, p. 13). Así, el alcance de un marco maestro es tal que logra unir diferentes marcos de distintos movimientos sociales, que pretenden hacerles frente a problemas distintos con estrategias propias. En este sentido, dicen Snow y Benford (1992), un marco maestro posee mayor capacidad de atribución y mayor potencia movilizadora. Es decir, en cuanto reúne a distintos movimientos sociales, logra generar más acciones colectivas de las que originaría un solo movimiento por separado.

Un ejemplo de un marco maestro es el marco de los derechos humanos al que apela la red en pro del envejecimiento (Sciubba, 2014). En 
esta red se desarrollan distintos marcos que abogan por dar prioridad a las personas mayores; allí, la diversidad de marcos juega a favor de la red, en virtud del marco maestro de los derechos humanos que puede rastrearse por medio de los marcos de diagnóstico, pronóstico y motivacional que conforman la red. Otro ejemplo de un marco maestro es el de la no violencia al cual se inscribe el Movimiento Lhakar. Este movimiento promueve que, cada miércoles, la población tibetana utilice el chupa (prenda tradicional del Tíbet) como rechazo a la represión ejercida por el gobierno chino en el territorio tibetano. Así, según muestra Dicky Yangzom (2016), este movimiento actúa bajo el marco maestro de la no violencia; que es el mismo que evoca el ahimsa promulgado por Gandhi, la idea de la desobediencia civil de Martin Luther King, y las enseñanzas del budismo tibetano. De acuerdo con esto, es posible comprender la manera en la que un marco maestro puede cobijar, a la manera de paradigma, distintos marcos particulares, para hacer más fuertes a las distintas acciones colectivas que se dan en su nombre.

En suma, un marco de acción colectiva, sea común o maestro, organiza las creencias, valores, planes y campañas de los movimientos sociales con el fin de lograr distintas acciones colectivas para dar solución a una serie de problemas. Ahora bien, ¿cómo se garantiza el éxito de un marco en términos de movilización social? Snow y Benford (1988) utilizan el término resonancia de marco para responder a esta pregunta. En su opinión, si existe una buena alineación entre un marco propuesto y un posible participante del movimiento, es porque tal marco "resuena" en esta persona. En sus palabras, tal marco "toca un acorde sensible". Esta metáfora musical invita a los investigadores a examinar cuáles (y de qué tipo) son -o deben ser- las condiciones o características de los marcos para que resuenen en los posibles participantes del movimiento social.

La resonancia de marco es un medidor para comprender la potencia movilizadora de un movimiento. Es decir, a mayor resonancia, más potente es el marco. Snow y Benford (1992) exponen tres variables para comprender dicho medidor: credibilidad empírica, conmensurabilidad experiencial y fidelidad narrativa. La primera se refiere a las bases empíricas sobre las cuales se sustenta un enmarcado de diagnóstico; es decir, la evidencia con la cual se atribuye un 
responsable a un problema colectivo. La segunda atiende a la cercanía entre el problema a resolver y la vida cotidiana de las personas. Es decir, entre más cerca se esté del problema, más conmensurabilidad experiencial habrá con el marco propuesto por un movimiento. Por último, la fidelidad narrativa hace referencia a la cercanía existente entre las ideas promovidas por un marco y el sistema de creencias de una persona. Así, estas tres variables permiten comprender cómo y por qué un marco de acción colectiva resuena en una persona. En otras palabras, cómo y porqué una persona puede convertirse en un participante de un movimiento social.

\section{¿Cómo se crea un marco de acción colectiva?}

La exposición anterior introdujo la teoría del enmarcado de los estudios sobre los movimientos sociales. No obstante, Snow y Benford (1992) no exponen detalladamente cómo es que una SMO construye un marco, aun cuando caracterizan los procesos discursivo, estratégico y controvertido de enmarcado. Por ello, expondremos tres reflexiones referenciadas en la literatura sobre movimientos sociales que intentan comprender cómo sucede tal construcción.

En primer lugar, William Gamson y Kathryn Lasch (1983) afirman que los marcos son construidos y presentados mediante dispositivos simbólicos que comprimen la cultura de un asunto. Estos autores reconocen dos tipos de dispositivos: de enmarcado y de razonamiento. Los primeros son las metáforas, ejemplos, eslóganes, representaciones e imágenes usadas en un discurso, mientras que los segundos son las causas, consecuencias y apelaciones a principios con los que se justifica la acción promovida en un discurso. De este modo, la comprensión de tales dispositivos permite dilucidar la manera en la que se construye un marco. En segundo lugar, John Noakes y Hank Johnston (2005, p. 7) sostienen que "si reconocemos que los marcos son construidos desde una fábrica cultural y tienen un contenido específico, entonces podemos describir los 'materiales' de los cuales están hechos". En tal sentido, estos autores sostienen que la producción discursiva es el objeto que permite comprender los materiales constitutivos de un marco. En tercer lugar, Paul McLean (1998, p. 55) sostiene que "la cultura es mejor comprendida como un 
espacio cognitivo multidimensional en el que distintos marcos son desplegados". De esta manera, un marco determina cierta capacidad de acción dentro de un espacio cultural particular. Para comprender dicho espacio, es necesario prestar atención a las palabras clave, imágenes y frases concretamente construidas que evocan un marco en cuestión.

Las reflexiones anteriores comparten la idea según la cual la construcción de un marco se relaciona con la cultura en la que se inscribe la persona (o personas) que crea(n) o emplea(n) el marco en cuestión. Además, sostienen que la forma de comprender tal construcción es mediante elementos discursivos de distintas clases. No obstante, para el caso de los movimientos sociales, tales elementos son apreciables generalmente en su actuación pública, pues se muestran en discursos, campañas y manifiestos, entre otros recursos comunicacionales. Esto quiere decir que, aunque la producción discursiva nos puede dar indicios de la forma en la que se ha construido un marco de acción colectiva, indican la presencia del marco resultante de dicha construcción. Por tanto, las reflexiones presentadas fallan al momento de explicar el proceso de construcción de un marco, aun cuando exponen elementos valiosos que se han utilizado como referencia para distintas investigaciones empíricas sobre el enmarcado en movimientos sociales. En este sentido, si se quiere comprender el proceso de construcción de un marco de acción colectiva es necesario analizar las dinámicas internas de una SMO. Esta vía, no obstante, ha sido poco transitada por los teóricos en movimientos sociales. Tal como afirman Snow y Benford (1988):

[...] el lado más visible de los movimientos sociales es su lado público [...] Puesto que este es el lado que es capturado, catalogado y archivado por los medios de comunicación, y puesto que muchos investigadores hoy en día tienen una inclinación por examinar la acción colectiva por medio de tales fuentes de datos, no es de extrañar que sea el lado que aparece de manera más prominente en la literatura. Los movimientos sociales, sin embargo, tienen otra cara, que no se ha comprendido claramente. Es en este lado donde se encuentran los esfuerzos de enmarcado, las negociaciones y las disputas que [...] forman parte del trabajo significativo de tales movimientos (1988, p. 214).

Esta cita apoya la idea de que la construcción de un marco de acción colectiva sucede en el "espacio" intramovimiento, donde 
se producen los elementos discursivos que luego transmiten el marco construido. Es decir, en las interacciones comunicativas que los activistas tienen de manera privada. Además, estos autores mencionan procesos, distintos al enmarcado, que pueden dar luz sobre lo que sucede en este espacio: la negociación y la disputa. En adición a esto, Hunt et al. (1994, p. 193) sostienen que los procesos de enmarcado suceden "por medio de negociaciones e interacciones tras bambalinas".

Para comprender estos procesos es útil remitirse al trabajo de Benford (1993) sobre disputas de marco. Este autor reconoce que no necesariamente todas las personas comparten el mismo marco o interpretación de la realidad. En sus palabras: "[...] en estos casos 'la realidad' debe ser negociada. De la misma manera, los actores de los movimientos sociales a menudo se encuentran en la empresa de negociar lo que es real" (1993, p. 678). En concreto, una negociación ocurre cuando no se comparte la misma interpretación de la realidad.

Benford retoma el concepto de disputa de marco de Goffman (1974) para referirse a lo que ha denominado inicialmente negociación. En dicha línea, el autor reconoce tres tipos de "disputas de marco intramovimiento": el primero es el ya expuesto (negociación), que versa sobre distintas interpretaciones de la realidad. En este caso lo que está en juego es el enmarcado de diagnóstico, es decir, la pregunta por cuál es el problema que constituye la razón de ser del movimiento. El segundo tipo de disputa es acerca de visiones dispares de una realidad alterna, esto es, acerca del enmarcado de pronóstico que determina cómo se debería transformar un aspecto problemático de la realidad. El último tipo de disputa se concentra en cómo presentar la realidad, y se corresponde con el enmarcado motivacional que construye los elementos discursivos de una campaña.

El estudio de Benford se enfoca únicamente en el análisis de aquello que denomina disputas de marco interorganizacionales, es decir, aquellas que ocurren entre dos SMO distintas. Así, su estudio de caso es el de las disputas ocurridas entre los movimientos sociales a favor del desarme nuclear de Estados Unidos a principios de la década de 1980. En este sentido, a pesar de la caracterización de las disputas intramovimiento, el autor se remite, nuevamente, al aspecto público de los movimientos sociales. 
Según lo expuesto, el trabajo de Benford es útil para comprender los procesos de negociación y disputa. A saber, una negociación es un tipo de disputa que se relaciona con el enmarcado de diagnóstico. Además, este autor muestra un elemento importante para comprender el proceso de construcción del enmarcado; esto es, que un marco de acción colectiva es el resultado de procesos comunicativos, entendidos como medios útiles para direccionar una acción colectiva. A pesar de ello, el análisis de Benford es insuficiente al momento de comprender las particularidades que envuelven tales disputas; por tanto, no ofrece una explicación completa de cómo se construye un marco de acción colectiva. En adición a esto, el autor no ofrece justificación alguna del porqué utilizar el término "negociación" para explicar la disputa sobre el enmarcado de diagnóstico. Por un lado, si este término es entendido como una metáfora, no es evidente que los activistas se comporten como negociantes a la hora de definir cuál es el problema (y el responsable) que enfrentará su $\mathrm{SMO}$, ni mucho menos que "la realidad" sea un concepto que se mida y negocie como una moneda.

Por otro lado, lo más probable es que el término "negociación" se refiera a un tipo de diálogo argumentativo. En este sentido, la taxonomía de diálogos argumentativos de Douglas Walton y Erik Krabbe (2017 [1995]) puede servir de base para analizar esta cuestión. Según estos autores, uno de los siete tipos ${ }^{3}$ de diálogos básicos es el de negociación. Este diálogo tiene cuatro características: (1) es ocasionado por un conflicto de intereses entre las partes o por la necesidad de cooperación, (2) su meta principal es cerrar un trato, (3) la meta de los participantes es beneficiarse individualmente del trato, y (4) puede producir beneficios colaterales como: llegar a un acuerdo, reforzar la confianza, revelar posiciones, influenciar a una audiencia, o adquirir prestigio. De estas características, la tercera es, según los autores, la más relevante para este tipo de diálogo. Es decir, si no se

3 Walton y Krabbe (2017) presentan una tipología -no exhaustiva- de distintos diálogos argumentativos que sirve como modelo normativo que dicta cómo se debe participar en un diálogo para que su meta sea alcanzada. Así, los modelos presentados no se entienden como los comportamientos reales de las personas, sino como juegos de diálogos donde los jugadores disponen de turnos, movimientos y estrategias para lograr distintas metas individuales o generales, y algunos beneficios adicionales. Los diálogos son: de persuasión, negociación, investigación, deliberación, búsqueda de información, erístico y mixto. Además, los autores muestran subtipos de cada diálogo, la situación inicial que los origina, la meta principal, la meta de los participantes y los beneficios colaterales que se pueden obtener en cada diálogo. 
busca un beneficio individual, no se está negociando. Tal objetivo se logra por un proceso de "regateo egoísta" en el que se pretende llegar a un acuerdo llamativo para las partes. No obstante, tal regateo no implica falta de cooperación; al contrario, una negociación puede construir confianza y buenos canales de comunicación entre las partes involucradas.

No es evidente que los activistas de una SMO estén inmersos en un diálogo de negociación cuando realizan un enmarcado de la acción colectiva puesto que tal enmarcado no se relaciona con las características de la negociación recién expuestas. Es decir, tal enmarcado (1) no es ocasionado por un conflicto de intereses o por la necesidad de cooperar, sino más bien por la necesidad de resolver un problema práctico-colectivo; (2) su meta principal no es cerrar un trato, sino resolver el problema colectivo; (3) la meta de los participantes (en este caso activistas) no es beneficiarse individualmente, sino ofrecer estrategias en pro de la resolución del problema, y (4) sí puede producir los beneficios colaterales de revelar posiciones, o adquirir prestigio. Sin embargo, los beneficios de influenciar a una audiencia y reforzar la confianza no ocurren en tal enmarcado, en cuanto los activistas no están expuestos a una audiencia, ni tienen razones para poner en cuestión su confianza. Esto, no obstante, no significa que los activistas no puedan beneficiarse individualmente de su participación en una $\mathrm{SMO}$, sino que no es la característica propia de un proceso de enmarcado de acción colectiva.

A pesar de que el enmarcado de diagnóstico no se corresponda con el diálogo argumentativo de negociación, la propuesta de Benford (1993) permite aventurar una hipótesis provisional: un marco de acción colectiva se construye por medio de un tipo de diálogo argumentativo. Esta hipótesis está apoyada por la literatura en deliberación pública, que hace acopio de la teoría de enmarcado bajo un principio básico: "[...] la comunicación es el único mecanismo de enmarcado" (Bodtker \& Jameson, 1997, p. 239). Tal principio se justifica mediante el argumento según el cual "el enmarcado es un proceso comunicativo en el que se estructura el contexto de significado [...] en el que se sostiene una deliberación" (Barisione, 2012, p. 1). En este sentido, el marco influencia los mensajes que se 
consideran apropiados en un contexto y, al mismo tiempo, es creado por dichos mensajes. En resumen, de acuerdo con estos autores, el enmarcado es un fenómeno esencialmente interactivo (Black, 2013).

\section{Enmarcado de acción colectiva y el diálogo deliberativo}

La pregunta consecuente con lo anterior es: ¿qué tipo de diálogo argumentativo es el enmarcado de una acción colectiva? Retomando la propuesta de Walton y Krabbe (2017), parece que el diálogo deliberativo es una opción prometedora para responder a esta pregunta. Este diálogo tiene cinco características: primero, es ocasionado por la necesidad de acción, en otras palabras, por un problema de carácter práctico frente al cual no se tiene claro cómo actuar para solucionarlo. Segundo, su meta principal es resolver el problema práctico-colectivo. Para lograr esto, los involucrados deben visualizar -de acuerdo con su presente- cómo podría cambiar el mundo si se decidieran a actuar de determinada manera; es decir, que deben hacer planes y sondeos de contingencias que permitan comprender las consecuencias de las acciones propuestas. Según los autores, esto hace que la deliberación sea un diálogo complejo, puesto que los involucrados no tienen control sobre el futuro acerca del cual están especulando. Por esto, las decisiones tomadas deben ser revisadas constantemente de acuerdo con el conocimiento de las partes y el desarrollo de las consecuencias de la acción.

Tercero, la meta de los participantes es influir en la decisión final; por esto, la discusión usualmente gira en torno a los medios disponibles para -o los fines pretendidos con- un curso de acción propuesto. No obstante, si un participante decide defender una propuesta particular de acción -en contra de otra propuesta- se daría paso a un subdiálogo persuasivo para discutir las propuestas en cuestión. Cuarto, el diálogo deliberativo puede producir beneficios colaterales tales como llegar a un acuerdo, expresar emociones, revelar posiciones o adquirir prestigio. Quinto, la argumentación central de este diálogo es el razonamiento práctico, esto es, un razonamiento dirigido a fines y basado en la consideración de medios de los que dispone un agente para decidir qué hacer y así cumplir su fin. Para 
resumir, un diálogo deliberativo es un tipo de diálogo argumentativo en el que sus participantes proponen y ponderan cursos de acción para resolver un problema práctico.

Ahora bien, si se quiere mostrar que el proceso de creación de un marco de acción colectiva sucede en un diálogo deliberativo, es necesario probar tres cosas: (1) que dicho marco es ocasionado por $-\mathrm{y}$ está dirigido a resolver- un problema práctico; (2) que los activistas pretenden influir en la decisión final y que su diálogo puede generar beneficios colaterales, y (3) que los activistas usan el razonamiento práctico para definir el marco. Antes de mostrar estas cuestiones, es necesario definir cuál esquema de razonamiento práctico se utilizará en el análisis, puesto que, desde la descripción inicial de Walton y Krabbe (2017), dicho esquema ha sido muy discutido.

Robert Audi (2006) propone un esquema del razonamiento práctico que tiene en cuenta los motivos y los medios considerados por un agente para definir cuál acción debería realizar, es decir, para hacer un juicio práctico (o juicio normativo, siguiendo a Isabela Fairclough y Norman Fairclough, 2012). Otros autores han agregado distintas partes al esquema inicial, amparados en la idea de que tal esquema es insuficiente para dar cuenta de todas las razones que preceden al juicio, así como para dar cuenta de la forma en la que, de hecho, sucede una argumentación práctica. Así, Walton (2007) propone un esquema que considera los valores a los que apelan los agentes para realizar un juicio práctico. Además, Macagno y Walton (2018) sostienen que el razonamiento práctico no se corresponde siempre y exclusivamente con un mismo tipo de argumento práctico, a pesar de que todo argumento práctico representa la fase deliberativa de una toma de decisiones. Teniendo en cuenta esta discusión, se utilizará aquí el esquema propuesto por Isabela Fairclough y Norman Fairclough (2012) para trazar una relación entre el proceso de enmarcado de la acción colectiva y el diálogo deliberativo. Tal elección atiende a que ese esquema tiene los elementos suficientes para trazar dicha relación. Estos autores sostienen que, tanto el fin como las circunstancias del agente, son determinados por sus valores, lo cual repercute en su juicio práctico. El esquema argumentativo se podría expresar así: 
Premisa circunstancial: Dadas las circunstancias C del agente Premisa teleológica: Dado el fin $F$ del agente

Premisa axiológica: Dado los valores $V$ del agente que determinan $F$ y $C$

Premisa instrumental: Dado que hacer $A$ es suficiente o necesario para $F$

Juicio normativo: El agente (presuntamente) debería hacer $A$

Una vez escogido el esquema de razonamiento práctico, es posible afirmar que el enmarcado de acción colectiva sí sucede en un diálogo deliberativo como el propuesto por Walton y Krabbe (2017). Esto es: (1) un marco de acción colectiva es ocasionado por la necesidad de emprender una acción colectiva que pretende resolver un problema colectivo; (2) la meta de cada uno de los activistas al construir el marco es influir en la decisión final; (3) el proceso de creación del marco puede generar beneficios colaterales, como llegar a un acuerdo, expresar emociones, revelar posiciones, o adquirir prestigio, y (4) el proceso de enmarcado corresponde al razonamiento práctico. Esto puede explicarse si se entiende, por un lado, que, las premisas circunstancial y axiológica pueden relacionarse con el enmarcado de diagnóstico, en cuanto que es allí donde se identifican las circunstancias problemáticas para un colectivo, así como los valores compartidos por dicho colectivo. Por otro lado, que, las premisas teleológica e instrumental se relacionan con el enmarcado de pronóstico, dado que definen el fin, que es resolver el problema diagnosticado y una propuesta de solución apropiada. Por eso, el resultado de estos procesos de enmarcado no es otro que el marco de acción colectiva, entendido como un juicio práctico que dicta las acciones que componen una campaña de movilización social.

Como ha de notarse, la clasificación anterior deja por fuera al enmarcado motivacional. Esto se debe a que tal proceso se considera aquí como un efecto del marco, no como parte del marco mismo. Es decir, el enmarcado motivacional ofrece motivos para actuar: esto implica que la acción ya ha sido previamente definida, mediante un proceso análogo al razonamiento práctico. Basta entonces con el diagnóstico y el pronóstico para que haya un marco de acción colectiva, puesto que los activistas involucrados pueden, por alguna 
razón, no ejecutar la campaña planeada y, en esta medida, no realizar un enmarcado motivacional de manera pública. Ello no significa, sin embargo, que el marco de acción colectiva esté incompleto, sino tan solo que no ha sido ejecutado.

\section{Conclusión}

Este artículo ha intentado elucidar el tipo de acción relacionada con la organización que realizan los activistas de una SMO. Para ello, el análisis se centró en la teoría del enmarcado de los estudios sobre movimientos sociales. De este modo, en la primera parte se expuso lo que es un marco de acción colectiva, y en la segunda se examinaron críticamente distintas explicaciones relevantes acerca de cómo se crea un marco de acción colectiva. Tal examen fue útil para centrar una explicación del proceso de enmarcado en el ámbito intramural de las $\mathrm{SMO}$. Así, se examinó también el análisis de disputas de marco de Benford (1993) y se determinó la inviabilidad de la utilización del término "negociación" para describir el proceso de enmarcado. En este sentido, el análisis de la descripción del diálogo deliberativo de Walton y Krabbe (2017) permitió concluir, mediante una interpretación distinta del proceso de enmarcado, que la creación de un marco de acción colectiva sucede en un diálogo deliberativo.

El recuento anterior permite apreciar que el trabajo realizado puede aportar a los estudios sobre los movimientos sociales, al menos en dos sentidos: (1) responde a la cuestión, insatisfactoriamente resuelta, sobre cómo se crea un marco de acción colectiva, al tiempo que complementa la idea de disputas de marco intramovimiento, y (2) expone una nueva forma de comprender las tareas básicas del enmarcado, que es importante para apreciar el carácter deliberativo del proceso de enmarcado. Ahora, ¿cómo aporta este trabajo a una reflexión general sobre la acción colectiva?

Al afirmar que el enmarcado de acción colectiva se realiza dentro de un diálogo deliberativo se está afirmando que los activistas realizan una acción colectiva deliberada. De acuerdo con Júlder Gómez (2019, p. 1), este tipo de acciones "se ejecutan como realización de una decisión grupal a la que se ha arribado en un diálogo argumentativo, en un diálogo deliberativo". Para este autor, tales acciones se entienden 
como la conclusión de una deliberación en la que sus participantes tienen "el compromiso con la argumentación como procedimiento para la elección de un curso de acción resolutivo de un problema práctico y con la decisión colectiva en cuanto condición apremiante para la realización de este curso de acción” (Gómez, 2019, p. 17).

El concepto de acción colectiva deliberada de Gómez apela a los compromisos que asumen los hablantes durante un diálogo argumentativo. Por esta razón, tal acción podría ser constatable empíricamente en el caso en el que sea la ejecución de una conclusión de un diálogo deliberativo considerado por los participantes como un procedimiento útil para elegir cursos de acción. Así, si la hipótesis de que el enmarcado de acción colectiva sucede dentro de un diálogo deliberativo es correcta, debería poder constatarse que un marco de acción colectiva es la conclusión de una deliberación, así como que tal diálogo es reconocido por los activistas como un procedimiento para elegir cursos de acción.

Este concepto de acción colectiva deliberada está formulado para el contexto de una deliberación simple. No obstante, las dinámicas de una $\mathrm{SMO}$ no suceden de esta forma, puesto que el enmarcado es establecido mediante más de un diálogo en los que no necesariamente se encuentran reunidos los mismos activistas cada vez. Esto no implica que el proceso de enmarcado no pueda entenderse como una acción colectiva deliberada, siempre que se comprenda el contexto institucional en el que se inscribe. Es decir, el enmarcado de acción colectiva sucede bajo un contexto institucional (SMO) que logra comprometer a sus miembros con el cumplimiento de los compromisos adquiridos, así no estén presentes en la deliberación. Lo que sí se requiere para que la acción colectiva de los activistas sea entendida como deliberada es que estos logren concluir juntos cuál es el marco de acción colectiva a utilizar.

De acuerdo con lo anterior, al anclar la teoría del enmarcado con la teoría de la argumentación práctica, este artículo provee dos nuevas categorías de análisis para comprender la acción colectiva: el diálogo argumentativo y el razonamiento práctico. La primera determina el contexto en el que se planifica la acción, mientras que la segunda explica la forma en la que los agentes razonan al momento de actuar colectivamente. Esto es importante porque 
tales categorías permitirían diferenciar distintos tipos de acciones colectivas, así como comprender a distintos tipos de agentes involucrados en dichas acciones $\mathbf{I}$

\section{Referencias}

Audi, R. (2006). Practical Reasoning and Ethical Decision. Routledge.

Barisione, M. (2012). Framing a Deliberation: Deliberative Democracy and the Challenge of Framing Processes. Journal of Public Deliberation, 8(1), Art. 2. https://bit.ly/2QijZpN

Bateson, G. (1955). A theory of play and fantasy. Psychiatric Research Reports, 2, 39-51. https://bit.ly/2xHd5sH

Benford, R. D. (1993). Frame Disputes within the Nuclear Disarmament Movement. Social Forces, 71(3), 677-701. https://doi.org/10.1093/ sf/71.3.677

Benford, R. D. \& Snow, D. (2000). Framing Processes and Social Movements: An Overview and Assessment. Annual Review of Sociology, 26, 611639. https://doi.org/10.1146/annurev.soc.26.1.611

Black, L. W. (2013). Framing Democracy and Conflict Through Storytelling in Deliberative Groups. Journal of Public Deliberation, 9(1), Art. 4. https://bit.ly/3d6zU9x

Bodtker, A. M. \& Jameson, J. K. (1997). Mediation as Mutual Influence: Reexamining the Use of Framing and Reframing. Mediation Quarterly, 14(3), 237-249. https://doi.org/10.1002/crq.3900140307

Diani, M. (1995). Green Networks: A Structural Analysis of the Italian Environmental Movement. Edinburgh University Press.

Fairclough, I., \& Fairclough, N. (2012). Political Discourse Analysis: A method for advanced students. Routledge.

Gamson, W. A. \& Lasch K. E. (1983). The political culture of social welfare policy. En S. E. Spiro, \& E. Yuchtman-Yaar (Eds.), Evaluating the Welfare State: Social and Political Perspectives (pp. 397-415). Academic Press.

Goffman E. (1974). Frame Analysis: An Essay on the Organization of the Experience. Harper Colophon. 
Gómez, J. A. (2019). Diálogos deliberativos como explicación de acciones colectivas. Artículo no publicado.

Harré, R. (2006). El frame analysis de Goffman. En E. Goffman, Frame análisis: Los marcos de la experiencia (pp. XIII-XVI). Centro de Investigaciones Sociológicas, Siglo XXI.

Hunt, S. A., Benford, R. D. \& Snow, D. A. (1994). Identity Fields: Framing Processes and the Social Construction of Movement Identities. En E. Laraña, H. Johnston, \& J. R. Gusfield (Eds.), New Social Movements: From ideology to Identity (pp. 185-208). Temple University Press.

Macagno, F. \& Walton, D. N. (2018). Practical Reasoning Arguments: A Modular Approach. Argumentation, 32, 519-547. https:/doi. org/10.1007/s10503-018-9450-5

McLean, P. D. (1998). Frame Analysis of Favor Seeking in the Renaissance: Agency, Networks, and Political Culture. American Journal of Sociology, 104(1), 51-91. https://doi.org/10.1086/210002

Morris, A. D. \& Staggenborg, S. (2004). Leadership in Social Movements. En D. A. Snow, S. A. Soule, \& H. Kriesi (Eds.), The Blackwell companion to social movements (pp. 171-196). Blackwell Pub.

Noakes, J, A. \& Johnston, H. (2005). Frames of Protest: A Road Map to a Perspective. En J, A. Noakes, \& H. Johnston (Eds.), Frames of Protest: Social Movements and the Framing Perspective (pp. 1-32). Rowman \& Littlefield.

Rosch, E. (1973). Natural categories. Cognitive Psychology, 4(3), 328-350. https://doi.org/10.1016/0010-0285(73)90017-0

Sciubba, J. D. (2014). Framing and Power in Aging Advocacy. Social Movement Studies, 13(4), 465-481. https://doi.org/10.1080/14742837.20 13.860026

Snow, D. A. \& Benford, R. D. (1988). Ideology, frame resonance, and participant mobilization. International Social Movement Research, 1, 197-218. https://bit.ly/3aWvoIx

Snow, D. A. \& Benford, R. D. (1992). Master Frames and Cycles of Protest. En A. D. Morris \& C. M. Mueller (Eds.), Frontiers in Social Movement Theory (pp. 133-155). Yale University Press. 
Snow, D. A. \& Benford, R. D. (2000). Framing Processes and Social Movements: An Overview and Assessment. Annual Review of Sociology, 26, 611-639. https://bit.ly/38Tqgnh

Tilly, C. y Wood, L. J. (2009). Los movimientos sociales, 1768-2008: Desde sus orígenes a Facebook. Crítica.

Walton, D. N. (2007). Evaluating Practical Reasoning. Synthese, 157(2), 197-240. https://doi.org/10.1007/s11229-007-9157-x

Walton, D. N. \& Krabbe, E. C. W. (2017 [1995]). Argumentación y normatividad dialógica: Compromisos y razonamiento interpersonal (C. Santibáñez, Trad.). Palestra.

Willems, J. \& Jegers, M. (2012). Social Movement Structures in Relation to Goals and Forms of Action: An exploratory model. Canadian Journal of Nonprofit and Social Economy Research, 3(2), 67-81. https:// doi.org/10.22230/cjnser.2012v3n2a128

Williams, R. H. (2004). The Cultural Contexts of Collective Action: Constraints, Opportunities, and the Symbolic Life of Social Movements. En D. A. Snow, S. A. Soule \& H. Kriesi (Eds.), The Blackwell companion to social movements (pp. 91-115). Blackwell Pub.

Wittgenstein, L. (2008 [1953]). Investigaciones filosóficas. Crítica.

Yangzom, D. (2016). Clothing and social movements: Tibet and the politics of dress. Social Movement Studies, 15(6), 622-633. https://doi.org/10.10 80/14742837.2016.1213163 